\title{
TRATAMENTO PALIATIVO DO ADENOCARCINOMA GÁSTRICO
}

\author{
Palliative treatment of gastric adenocarcinoma \\ Fernando de Oliveira SOUZA, Luis Carlos Moreira ANTUNES, Luis Humberto Ribas dos SANTOS
}

Trabalho realizado no Departamento de Cirurgia e Serviço de Oncologia e Radioterapia do Hospital Universitário da Universidade Federal de Santa Maria, Santa Maria, RS, Brasil.

DESCRITORES - - Estômago. Adenocarcinoma. Tratamento paliativo. Cirurgia. Quimioterapia.

\section{Correspondência:}

Fernando de Oliveira Souza, e-mail:fsouza@via-rs.net

Fonte de financiamento: não há Conflito de interesses: não há

Recebido para publicação: 27/07/2010 Aceito para publicação: 25/01/2011

HEADINGS - Stomach. Adenocarcinoma. Paliative care. Surgery. Drug therapy.
RESUMO - Introdução: Embora decrescendo nos países do chamado primeiro mundo, o adenocarcinoma gástrico mantém-se como terceiro tumor mais frequente no sexo masculino mundialmente. Sua mortalidade é muito elevada, fruto do diagnóstico tardio em lesões muito avançadas, o que frequentemente torna paliativo seu tratamento, motivos pelos quais se justificam estudos no sentido de melhorar estes resultados. Método - Revisão da literatura através do portal de periódicos da CAPES indicados por busca no sites da Bireme e PubMed. Além disso, foram consultados os sumários do $8^{\circ}$ Congresso Internacional de Câncer Gástrico em 2009. Foi apresentada uma sugestão de algoritmo de atendimento destes pacientes. Conclusões - O surgimento de novas drogas anticancer, mais efetivas, está propiciando novas alternativas para a ressecção gástrica como tratamento paliativo. Novos protocolos estão surgindo mostrando boas perspectivas para melhorar os resultados desta doença.
ABSTRACT - Introduction - Although decreasing in the well developed countries, gastric adenocarcinoma still represents the third most common cancer in males worldwide. Its mortality is very high because of the lateness of its diagnosis over advanced lesions, which turns palliative its treatment in the majority of the cases. Method - Literature review using CAPES, PubMed and Bireme sites as well as the abstracts of the $8^{\circ}$ International Gastric Cancer Congress which was held in Krakow in 2009. Conclusions - The release of new anticancer drugs against gastric cancer is providing a revival of gastrectomy as an effective palliative treatment of advanced gastric cancer. New protocols are being published, showing better results in the treatment of this disease.

\section{INTRODUÇÃO}

E mbora decrescendo nos países do chamado primeiro mundo, o adenocarcinoma gástrico (AG) continua sendo dos mais frequentes no sexo masculino (ocupa a terceira posição depois de próstata e pulmão), porém sua importância é ainda maior em função de sua alta mortalidade (segunda posição depois de pulmão). Foram computados mundialmente no ano de 2008 quase um milhão de novos casos e 780 mil óbitos $^{11}$.

No Brasil, esperam-se para 2010, aproximadamente 22000 novos casos considerando-se ambos os sexos, segundo estimativas do INCA ${ }^{20}$.

Analisando os resultados obtidos no tratamento do adenocarcinoma gástrico no mundo ocidental, pode-se considerá-lo de uma maneira geral como paliativo, visto que as recidivas são o seguimento mais provável e a sobrevida em cinco anos atinge em média somente $20 \%$, computando-se todos os estágios em conjunto.

Mesmo as ressecções de residualidade zero (R0), com intenção curativa, conforme proposto por Hermanek ${ }^{24}$ em 1994, ou seja, margens cirúrgicas microscopicamente negativas assim como o leito tumoral, a recidiva chega a 52\% ${ }^{4}$ a maioria loco-regional, seja linfonodos ou peritônio envolvido. Antecipadamente, já se pode visualizar a necessidade de estabelecer critérios adicionais para a definição de operação com intenção curativa já que, como foi visto, não é certo defini-la como tal com um índice tão elevado de recorrência 
da doença. Mais correto seria definir R0 como "tumor residual não detectável pelos métodos diagnósticos convencionais". Aintençãocurativa deveriaincluircitologia negativa de líquido peritoneal (5 a 20\% dos pacientes submetidos a ressecções "curativas" apresentam células neoplásicas no lavado peritoneal mesmo na ausência de lesões macroscópicas, principalmente em tumores $\mathrm{T}^{43}$ ), assim como pesquisa negativa de células isoladas tumorais no sangue periférico, portal (encontradas em 18 a $24 \%$ dos $\operatorname{casos}^{39}$ ) e na medula óssea dos pacientes (encontradas em $50 \%$ dos casos no pré-operatório ${ }^{36}$. Brennan e Karpeh ${ }^{12}$ consideram as ressecções gástricas em pacientes com tumores T3 como paliativas pela alta incidência de citologia positiva para células neoplásicas nestes casos.

Discorrer-se-á a seguir sobre o tratamento paliativo do ponto de vista cirúrgico, quimioterápico e suas associações, adicionando proposição de um algoritmo de atendimento (Figura 1), proposta esta que quer-se submeter à apreciação da comunidade científica, sempre tratando de tumores avançados.

\section{MÉTODO}

Foram utilizados os descritores "estômago" e "adenocarcinoma" para pesquisa no PubMed e Bireme e a seguir selecionadas as publicações pertinentes no Portal CAPES com atenção especial para metanálises e estudos clínicos controlados. Além disto, foi revisado o Abstract Book do $8^{\circ}$ Congresso Internacional de Câncer Gástrico realizado na Cracóvia, Polônia em 2009.

\section{Tratamento cirúrgico paliativo}

Do ponto de vista cirúrgico, a ressecção sempre que possível como tratamento paliativo deve ser analisada quanto a relação risco/benefício, entendendo-se como benefício a qualidade e quantidade da sobrevida destes pacientes. Para esta análise há que se particularizar cada caso baseando-se no grau de comprometimento provocado pela doença (estadiamento).

A primeira etapa no algoritmo de atendimento destes pacientes é a avaliação de sua aptidão em receber algum tipo de tratamento, seja cirúrgico, químio ou radioterápico. Para tanto, além dos exames de rotina, se adota escalas de performance como as de Karnofsky ${ }^{26}$ ou ECOG ${ }^{30}$. Os considerados não aptos são encaminhados para o melhor tratamento de suporte, os aptos para estadiamento clínico que inclui exame físico minucioso à procura de doença disseminada como, por exemplo, a plataforma de Blummer no toque retal, exames de imagem incluindo tomografia computadorizada de pelve, abdome (com contraste) e tórax, e marcadores tumorais no sangue (CEA e CA 19-9). A tomografia abdominal deve buscar evidências de envolvimento linfonodal satélite (linfonodos acima de $1 \mathrm{~cm}$ de diâmetro), metástases hepáticas e sinais de irresecabilidade como, por exemplo, invasão difusa do retroperitônio, com comprometimento de grandes vasos. O estudo gastroscópico com biópsia prévia idealmente deve vir acompanhado de ecoendoscopia.

Após esta avaliação inicial, são excluídos aqueles com sangramento tumoral ou obstrução gástrica significativos e os demais (principalmente os que apresentam tumores gástricos envolvendo todo o órgão e/ou linfoadenomegalia $>1 \mathrm{~cm}$ ) são encaminhados à laparoscopia com lavado peritoneal. A partir destes resultados, os pacientes com doença difusa (far advanced gastric cancer - FAGC), com um fator de incurabilidade, são encaminhados para ressecção gástrica de redução tumoral seguida de tratamento adjuvante. Aqueles com vários fatores de incurabilidade vão para radioquimioterapia paliativa. Os pacientes sem evidências de doença difusa (local advanced gastric cancer - LAGC), são indicados para tratamento quimioterápico perioperatório com três ciclos pré e três pós-operatórios. Estes pacientes devem ser monitorados na fase pré quanto à resposta à medicação

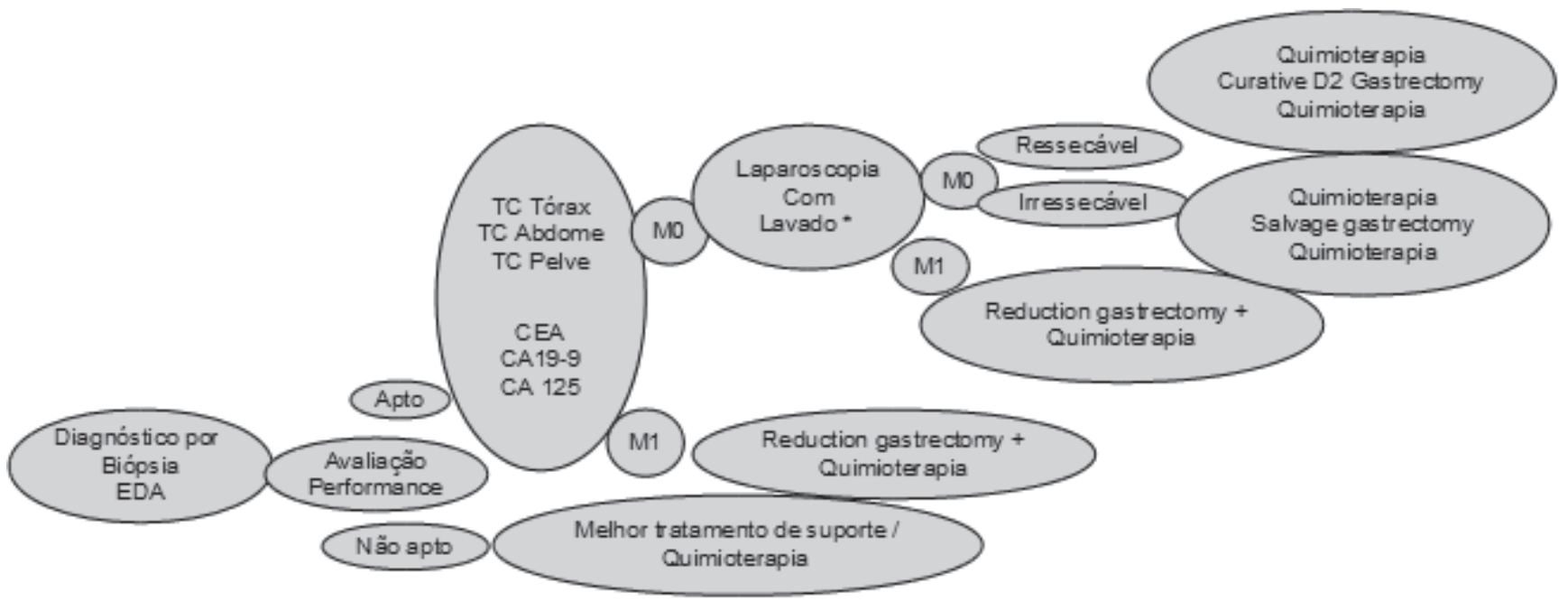

FIGURA 1 - Algoritmo proposto para melhor orientar o manuseio dos pacientes com câncer gástrico em tratamento paliativo 
utilizando os critérios recist ${ }^{19}$; no caso de não resposta, deve ser antecipado o seu encaminhamento para um procedimento cirúrgico.

A operação recomendada é a gastrectomia com linfadenectomia D2 respeitando-se as margens de segurança recomendadas para tumores difusos e intestinais e avaliação por congelação dos linfonodos para-aórticos (grupo 16). Nos pacientes com tumores considerados irressecáveis, seja no pré ou no transoperatório, desde que não haja doença difusa com vários fatores de incurabilidade, é recomendado tratamento neoajuvante na tentativa de redução tumoral e operação de revisão (2nd look) (Figura 1).

A ressecção multivisceral está indicada na ausência de doença difusa/sistêmica.

Todos estes procedimentos devem ser realizados em centros de referência com alto volume de casos no tratamento do câncer gástrico.

Comparando-se com a literatura atual, destacam-se como os pontos mais polêmicos do algoritmo - no que se refere ao tratamento cirúrgico paliativo do AG -, as gastrectomias de redução tumoral (reductive gastrectomy) para tratamento adjuvante em pacientes com um fator de incurabilidade, e as gastrectomias de resgate (salvage gastrectomy) após tratamento neoadjuvante em paciente inicialmente considerados irressecáveis.

Na primeira situação, foi ativado em fevereiro de 2008 um estudo randomizado controlado (RCT) entre Japão (JCOG 0705) e Coréia (KGC01) comparandose dois grupos de pacientes com AG estágio IV com um fator de incurabilidade, seja hepático, peritoneal ou linfonodal distante, um deles para tratamento exclusivamente quimioterápico e o outro encaminhado para gastrectomia de redução tumoral seguida do mesmo esquema quimioterápico com expectativa de $10 \%$ de incremento de sobrevida em dois anos no grupo cirúrgico ${ }^{38}$. Efetivamente, já em 2006, Saidi et al ${ }^{34}$ relataram aumento de sobrevida de 10,4 meses no grupo de pacientes operados concluindo que há um papel para o que eles chamaram de gastrectomia paliativa nos pacientes com AG estágio IV.

Quanto às gastrectomias de resgate, com a evolução e a elaboração de novas drogas anticancer, foram surgindo trabalhos propondo, nos pacientes com AG disseminados ou irressecáveis, operações de ressecção após tratamento neoadjuvante com resultados bastante satisfatórios ${ }^{14,25,44}$.

\section{Tratamento quimioterápico paliativo}

Câncer gástrico metastático: suporte clínico vs tratamento quimioterápico combinado

Em 1977, Glimelius, et al. ${ }^{23}$ publicaram um estudo randomizado que revelou maior taxa de sobrevida global com melhor qualidade de vida para os pacientes que receberam quimioterapia paliativa, comparado com tratamento de suporte clínico (69\% VS 47\%; $p<0,05)^{23}$. Entretanto, pelo menos três estudos da mesma década não confirmaram estes resultados ${ }^{18,28,33}$.
Com a evolução tanto dos quimioterápicos quanto dos cuidados de suporte, novos estudos realizados na década de 90 revelaram claro benefício a favor da quimioterapia paliativa. Metanálise publicada por Wagner, et al. ${ }^{41}$ com três estudos incluindo 184 pacientes, demonstrou HR de 0,39(IC:95\%; 0,28a 0,52) em relação a sobrevida global, a favor da quimioterapia.

Quimioterapia com agente único vs regime combinado Fluorouracil (5FU) foi o mais extensivamente agente quimioterápico estudado para câncer gástrico metastático, sendo usado como monoterapia em 10 estudos, e em nove regimes de quimioterapia combinada, de um total de 11 estudos revisados por Wagner, et al. ${ }^{41}$. Com 1472 pacientes comparando quimioterapia com um único agente vs combinação, os autores encontraram HR de 0,83 (IC: 95\%, 0,74 à 0,93) para sobrevida a favor de regimes de combinação, correspondendo a ganho de sobrevida de aproximadamente um mês. Seis destes estudos, que usaram 5FU como monoterapia, reportaram taxas de morte relacionada ao tratamento de $1,7 \%$ para o tratamento combinado e $0,8 \%$ para $5 \mathrm{FU}$ como agente único ${ }^{41}$.

Cisplatina e antracíclicos em regimes combinados de quimioterapia

A metanálise de Wagner, et al. ${ }^{41}$ avaliou também três estudos randomizados com 501 pacientes, comparando combinação de cisplatina/5FU com ou sem antracíclicos, demonstrando beneficio estatisticamente significativo a favor do regime com três drogas ( $\mathrm{HR}=$ 0,77; IC 95\%, 0,62 -0,91), com ganho de sobrevida global de aproximadamente dois meses. Esta revisão também avaliou o papel da cisplatina em combinações com três drogas, com sete estudos comparando 5FU/ antracíclico/cisplatina vs 5FU/ antracíclico, verificando ganho de sobrevida global de aproximadamente um mês (HR: 0,83; IC 95\%, 0,76-0,91)

$O$ regime ECF (epirrubicina, cisplatina e 5FU em infusão contínua) foi comparado com PELF, uma combinação de quatro drogas (epirrubicina, cisplatina, leucovorin e $5 \mathrm{FU}$ em bolus) que resultou em taxa de morte relacionada ao tratamento de $3,3 \%$ vs $0,6 \%$ (OR= 5,36 IC 95\%, 1,1 a 27,4; $p=0,028$ ), sugerindo aumento da toxicidade para o PELF. Outros dois estudos analisaram qualidade de vida, demonstrando superioridade para o ECF em relação ao 5FU/doxorrubicina/metotrexate e mitomicina/cisplatina/5FU.

Oxaliplatina e capecitabina nos regimes
combinados de quimioterapia
Cunningham, et al ${ }^{16}$ apresentaram os dados de um estudo fase III (REAL-2) com 1002 pacientes randomizados em quatro braços em formato $2 \times 2$, tendo o ECF como braço de referência, e avaliando a não inferioridade da substituição do 5FU pela capecitabina e da cisplatina pela oxaliplatina. A capecitabina não foi inferior ao $5 \mathrm{FU}$ (HR: 0,86; IC:95\%, 0,80-0,99) e a oxaliplatina não foi 
inferior à cisplatina (HR: 0,92; IC: 95\%, 0,80-1,10). O grupo que recebeu o regime EOX (epirrubicina/oxaliplatina/ capecitabina) apresentou maior sobrevida em um ano $(46,8 \%$ vs $37,7 \%, p=0,02)$ e sobrevida global $(11,2$ vs 9,9 meses, $p=0,02$ ) quando comparado ao ECF. Os quatro regimes apresentaram taxas de resposta semelhantes, sem diferença estatística significativa com 47,9\% para EOX , 46,4\% para EOF (epirrubicina, oxaliplatina e 5FU), $42,4 \%$ para ECX (epirrubicina, cisplatina e capecitabina) e $40,7 \%$ para ECF. Os regimes contendo oxaliplatina foram bem tolerados, apresentando menor incidência de neutropenia severa, alopécia e nefrotoxicidade, mas com maiores taxas de neuropatia periférica e diarréia ${ }^{16}$.

Outro estudo fase III, com 220 pacientes, comparou um regime contendo oxaliplatina (FLO: oxaliplatina/ 5FU/leucovorin) versus um regime com cisplatina (FLP: cisplatina/5FU/leucovorin) em relação ao sobrevida livre de progressão(SLP) com base na análise de intenção de tratar. Foi encontrada uma tendência em relação ao TLP para o regime $\mathrm{FLO}$, embora sem diferença estatística significativa (5,8 versus 3,9 meses; $p=0,077)$, assim como para a sobrevida global (10,7 versus 8,8 meses). Entretanto, quando analisados pacientes acima de 65 anos $(n=94)$, o regime FLO revelou-se significativamente superior em relação à taxa de resposta $(41,3$ versus $16,7 \% ; p=0,012)$, tempo para falha do tratamento $(5,4$ versus 2,3 meses; $\mathrm{P}<0,001)$, e SLP $(6,0$ versus 3,1 meses; $p=0,029$ ), além de um aumento da sobrevida global (13,9 versus 7,2 meses). Este grupo também apresentou menor toxicidade quanto à leucopenia grau 1-4, náusea, fadiga e nefrotoxicidade, porém maior incidência de neuropatia periférica ${ }^{7}$.

\section{Docetaxel}

O uso do docetaxel em primeira linha para câncer gástrico avançado foi investigado no estudo TAX 325, cuja fase II comparou os regimes docetaxel/cisplatina (DC) e docetaxel/cisplatina/5FU (DCF) com o objetivo de identificar o regime mais efetivo. O DCF foi superior em eficácia ( $43 \%$ vs $26 \%$ ) e tempo de progressão (5,9 vs 5 meses), sendo escolhido para fase III. Para esta fase, foram randomizados 457 pacientes em dois grupos para receber primeira linha de DCF ou CF (cisplatina e 5FU). Novamente o DCF foi superior, com melhores resultados em relação ao tempo para progressão $(5,6$ vs 3,7 meses, $p=0,0004)$, sobrevida global $(9,2$ vs 8,6 meses, $p=0,02)$, sobrevida em dois anos ( $18 \%$ vs $9 \%$, taxa de resposta global (37 vs $25 \%, p=0,01)$ e análise de qualidade de vida. Entretanto, a toxicidade foi significativamente superior, com taxas de neutropenia grau III e IV de $82,3 \%$, e incidência de neutropenia febril de $30 \%$ no grupo DCF, devendo-se monitorizar cuidadosamente as reações adversas deste regime $e^{40,4,5}$.

\section{Irinotecano}

O estudo V-306 avaliou o uso de irinotecano em regime de combinação para primeira linha de câncer gástrico avançado em um estudo inicialmente fase II que comparou IC (irinotecano e cisplatina) e ILF (irinotecano, leucovorin e fluorouracil), definindo a superioridade deste último ${ }^{13}$. O estudo fase III do V-306, comparou o ILF com CF, e demonstrou tendência a maior tempo para progressão ( 5 vs 4,2 meses, $p=0,088$ ) e taxa de resposta $(31,8 \%$ vs $25,8 \%)$ a favor do IFL, mas sem diferença estatística significativa. Com tempo de sobrevida média similar nos dois grupos ( 9 vs 8,7 meses, $p=0,53$ ), mas menor toxicidade para o grupo com IFL, este pode ser usado como alternativa ao regime $\mathrm{CF}^{17}$. Bouche, et al. ${ }^{10}$ publicaram um estudo fase II comparando outro regime com irinotecano, combinado a leucovorin, $5 \mathrm{FU}$ em bolus e 5FU em infusão continua (FOLFIRI), com LF (leucovorin e 5FU) ou CLF (cisplatina, leucovorin e 5FU). As taxas de resposta objetiva, TP e SG foram superiores com o regime FOLFIRI ${ }^{10}$.

\section{S-1}

O uso de uma quarta geração de fluoropirimidinas chamada de S-1 e composta por tegafur/ gimeracil/ oteracil tem sido investigado principalmente no Japão, onde foi aprovado como quimioterapia paliativa ou tratamento adjuvante para pacientes submetidos à ressecção curativa. Outros países asiáticos também definiram regimes com S-1 como adjuvância (Coréia) ou como tratamento padrão em câncer gástrico avançado (Coréia, Singapura e China). O SPIRITS, um estudo fase III realizado por Koizumi, et al. ${ }^{29}$ com 305 pacientes, comparou S-1 sozinho com S-1/cisplatina, encontrando superioridade para o regime combinado em relação à sobrevida global ( $p=0,04 ; H R=0,77$, IC:95\%, 0,61-0,98) e à taxa de resposta (54\% vs $31 \%$ ). Apesar da maior toxicidade (maior incidência de neutropenia, anemia, náusea e anorexia) do regime combinado, este foi geralmente bem tolerado ${ }^{29}$. Outro estudo Japonês fase III, publicado por Boku et al. ${ }^{9}$, demonstrou sobrevida global similar para o S-1 quando comparado a 5FU ou à combinação irinotecano/cisplatina (tempos de sobrevida média de 11,4 vs 10,8 vs 12,3 meses) ${ }^{9}$.

Como a taxa de conversão metabólica da pródroga oral, tegafur para o fluorouracil parece ser diferente conforme a população étnica, foi conduzido um estudo fase I para estabelecer a dose máxima tolerada de S-1/cisplatina no ocidente ${ }^{3}$. Após avaliada a eficácia e segurança desta combinação, em um estudo multicêntrico fase II, Ajani , et al. ${ }^{6}$ publicaram os resultados do estudo FLAGS que incluiu 24 países e 146 centros, com 1053 pacientes estratificados (centro, número de sítios metastáticos, quimioterapia adjuvante prévia e extensão da doença) e randomizados para receber S-1/cisplatina ou 5FU/cisplatina (5FU em infusão de 24h). O regime S-1/cisplatina não apresentou aumento estatisticamente significativo da sobrevida global (8,6 vs 7,9 meses, com HR: 0,92; IC 95\%, 0,80 a 1,05; $p=0,20)$, mas resultou em menor toxicidade com taxas inferiores de neutropenia grau 3/4 (32,3 \% vs 63,6\%), neutropenia complicada ( $5 \%$ vs $14,4 \%)$, estomatite ( $1,3 \%$ vs $13,6 \%)$, hipocalemia (3,6\% vs $10,8 \%$ ) e morte relacionada ao tratamento $(2,5 \% \text { vs } 4,9 \% ; \mathrm{p}<0,05)^{6}$. 


\section{Anticorpo monoclonal}

Foi aprovado na Europa o uso do trastuzumabe combinado a quimioterapia para tratamento de primeira linha em tumores gástricos avançados positivos para HER2 ${ }^{37}$. O trastuzumabe é um anti-corpo monoclonal que bloqueia o receptor HER2, envolvido no crescimento celular. Um estudo fase III, multicêntrico, internacional e randomizado (ToGA) encontrou redução no risco de morte de $26 \%$ para os pacientes que receberam esta droga combinada à quimioterapia (5FU ou capecitabina em combinação com cisplatina) em comparação à quimioterapia apenas. A sobrevida global média encontrada foi de 13,8 meses no grupo do trastuzumabe vs 11,1 meses no grupo controle, tornando este 0 primeiro anti-corpo monoclonal a mostrar benefício na sobrevida entre as várias outras terapias alvo que estão sendo testadas para câncer gástrico ${ }^{31}$.

\section{Câncer gástrico localmente avançado}

A definição de câncer gástrico localmente avançado possui diferentes interpretações dependendo do autor e da instituição. Pode ser considerado como o tumor primário que o cirurgião não espera ressecar com margens negativas microscopicamente (sem possibilidade de ressecção curativa conforme exploração cirúrgica ou avaliação pré-operatoria com tomografia computadorizada, ultrassonografia endoscópica, laparoscopia, ou outros exames de imagem; recidivas locais sem evidência de metástases). Ou ainda, podese incluir doença completamente ressecável, mas com fatores de alto risco para recorrência local ou metástases à distância (envolvimento nodal, extensão além da parede gástrica, ou ambos) ${ }^{1}$.

Pacientes com invasão profunda, localmente volumosos e irressecáveis devido à infiltração de estruturas adjacentes, recebiam apenas tratamento quimioterápico paliativo. Entretanto, com a introdução da quimioterapia peri-operatória este cenário vem sendo modificado. A quimioterapia neo-adjuvante pode reduzir o volume tumoral, tornando o paciente operável; evitar o retardo no tratamento de possíveis micrometástases; melhorar a tolerabilidade à quimioterapia, uma vez que a quimioterapia adjuvante pode apresentar maior toxicidade devido ao alto consumo de energia e possíveis complicações pós-operatórias; e ainda, testar a resposta do paciente à determinada droga, definindo 0 regime empregado no pós-operatório ${ }^{45}$.

Schumacher, et al. ${ }^{35}$ publicaram em 2001 os resultados de cinco anos de seguimento de um estudo fase II com 42 pacientes com câncer gástrico localmente avançado (estágios IIIA, IIIB e IV). Estes foram inicialmente avaliados com endoscopia, ultrassonografia endoscópica, tomografia computadorizada para excluir infiltração de órgãos adjacentes e detecção de metástases à distância, e com laparoscopia para excluir carcinomatose peritoneal oculta. Cada paciente recebeu três ou quatro ciclos de quimioterapia combinada (doxorrubicina/cisplatina/etoposide). Após reavaliação,
36 pacientes foram submetidos à gastrectomia total, obtendo ressecção patológica completa em 31 (73,8\%). A sobrevida média global dos 42 pacientes foi de 19,1 meses, sendo que aqueles que não receberam tratamento cirúrgico, a sobrevida foi de apenas 1,5 meses, contra 22,2 meses para os submetidos ao tratamento cirúrgico (7,6 meses com ressecção incompleta vs 28,4 meses para ressecção completa, $p=0,0001)$. Ressalta-se superior taxa de sobrevida associada à maior resposta clínica à quimioterapia, com sobrevida média de 45 meses ${ }^{35}$. Vários outros estudos demonstraram benefício para quimioterapia neo-adjuvante no câncer gástrico localmente avançado, com aumento da ressecabilidade (42,4\%) e da sobrevida 2,21,22,27,32,42,44.

Em 2006, Cunningham, et al. ${ }^{15}$ publicaram os resultados do estudo MAGIC, onde 503 pacientes operáveis foram randomizados em um estudo fase III, para receber três ciclos de ECF antes e após a operação ou apenas tratamento cirúrgico. Os pacientes do grupo de quimioterapia peri-operatória apresentaram maiores taxas de $\mathrm{T} 1$ e T2 (51,7\% vs $36,8 \%)$ na análise patológica pós-operatória, e um ganho de 13\% na sobrevida em cinco anos (36\% vs $23 \%$ ), além de não ter ocorrido diferença estatística significativa na morbidade cirúrgica entre os dois grupos (45,7\% vs $45,3 \%)$. Houve maior número de operações consideradas curativas (79,3\% vs $70,3 \% ; p=0,003)$, aumento da sobrevida livre de progressão ( $H R=0,66$; IC95\%: 0,53 à 0,81) e da sobrevida global ( $H R=0,75$; IC95\%: 0,60 à 0,93 ) no braço com quimioterapia ${ }^{15}$.

Enquanto na doença metastática as doses dos regimes de quimioterapia não devem ser tão altas evitando-se toxicidades relevantes com intenção de melhorar a qualidade de vida com maior sobrevida -, por outro lado na doença localmente avançada a quimioterapia neoadjuvante tem como objetivo aumentar as taxas de ressecção e eventualmente de cura, devendo-se usar regimes combinados com duas ou três drogas e com doses menos flexíveis.

\section{CONCLUSÃO}

O surgimento de novas drogas anticancer, mais efetivas, está propiciando novas alternativas para a ressecção gástrica como tratamento paliativo. Novos protocolos estão surgindo mostrando boas perspectivas para melhorar os resultados desta doença.

REFERÊNCIAS

1. Abeloff MD: Clinical oncology, 3rd ed. Philadelphia, Pa.: Elsevier/ Churchill Livingstone, 2004.

2. Ajani JA, Mansfield PF, Lynch PM, Pisters PW, Feig B, Dumas P, Evans DB, Raijman I, Hargraves K, Curley S, Ota DM. Enhanced staging and all chemotherapy preoperatively in patients with potentially resectable gastric carcinoma. J Clin Oncol. 1999 Aug;17(8):2403-11.

3. Ajani JA, Faust J, Ikeda K, Yao JC, Anbe H, Carr KL, Houghton M, Urrea P. Phase I pharmacokinetic study of S-1 plus cisplatin in patients with advanced gastric carcinoma. J Clin Oncol 2005; 23(28): 695765. 
4. Ajani JA, Moiseyenko VM, Tjulandin S, Majlis A, Constenla M, Boni C, Rodrigues A, Fodor M, Chao Y, Voznyi E, Marabotti C, Van Cutsem E. Clinical benefit with docetaxel plus fluorouracil and cisplatin compared with cisplatin and fluorouracil in a phase III trial of advanced gastric or gastroesophageal cancer adenocarcinoma: the V-325 Study Group. J Clin Oncol 2007; 25(22): 3205-9.

5. Ajani JA, Moiseyenko VM, Tjulandin S, Majlis A, Constenla M, Boni C, Rodrigues A, Fodor M, Chao Y, Voznyi E, Awad L, Van Cutsem E. Quality of life with docetaxel plus cisplatin and fluorouracil compared with cisplatin and fluorouracil from a phase III trial for advanced gastric or gastroesophageal adenocarcinoma: the V-325 Study Group. J Clin Oncol 2007; 25(22): 3210-6.

6. Ajani JA, Rodriguez W, Bodoky G, Moiseyenko V, Lichinitser M, Gorbunova V, Vynnychenko I, Garin A, Lang I, Falcon S. Multicenter Phase III Comparison of Cisplatin/S-1 With Cisplatin/ Infusional Fluorouracil in Advanced Gastric or Gastroesophageal Adenocarcinoma Study: The FLAGS Trial. J Clin Oncol 2010;28(9):1547-53.

7. Al-Batran SE, Hartmann JT, Probst $S$, Schmalenberg $H$, Hollerbach $S$, Hofheinz R, Rethwisch V, Seipelt G, Homann N, Wilhelm G, Schuch G, Stoehlmacher J, Derigs HG, Hegewisch-Becker S, Grossmann J, Pauligk C, Atmaca A, Bokemeyer C, Knuth A, Jäger E. Phase III trial in metastatic gastroesophageal adenocarcinoma with fluorouracil, leucovorin plus either oxaliplatin or cisplatin: a study of the Arbeitsgemeinschaft Internistische Onkologie. J Clin Oncol 2008; 26(9): 1435-42.

8. Allum W, Garofalo A, Degiuli M, Schuhmacher C. The first european union network of excellence for gastric cancer conference, Rome, Italy, april 2008. Gastric Cancer 2009;12:56-65.

9. Boku N, Yamamoto S, Fukuda H, Shirao K, Doi T, Sawaki A, Koizumi W, Saito H, Yamaguchi K, Takiuchi H, Nasu J, Ohtsu A. Fluorouracil versus combination of irinotecan plus cisplatin versus $\mathrm{S}-1$ in metastatic gastric cancer: a randomised phase 3 study. Lancet Oncol 2009; 10(11): 1063-9.

10. Bouché $\mathrm{O}$, Raoul JL, Bonnetain $\mathrm{F}$, Giovannini $\mathrm{M}$, Etienne $\mathrm{PL}$, Lledo G, Arsène D, Paitel JF, Guérin-Meyer V, Mitry E, Buecher B, Kaminsky MC, Seitz JF, Rougier P, Bedenne L, Milan C. Randomized multicenter phase II trial of a biweekly regimen of fluorouracil and leucovorin (LV5FU2), LV5FU2 plus cisplatin, or LV5FU2 plus irinotecan in patients with previously untreated metastatic gastric cancer: a Federation Francophone de Cancerologie Digestive Group Study-FFCD 9803. J Clin Oncol 2004; 22(21): 4319-28.

11. Boyle P, Levin B. World cancer report. IARC publications 2008.

12. Brennan MF, Karpeh MS Jr. Surgery for gastric cancer. Seminars in Oncology 1996;23(3):352-9.

13. Bugat R: Irinotecan in the treatment of gastric cancer. Ann Oncol 2003; 14 Suppl 2: ii37-40.

14.Ciseł B., Lewicka M., Jazienicki M., Mielko J., Kurylcio A.,Wierzbicki R., Budny W., Sokoluk M., Polkowski W. Results of perioperative chemotherapy and second look operation in patients with advanced initially inoperable gastric carcinoma. Poster 275, 8th International Gastric Cancer Congress, Abstract Book:170.

15. Cunningham D, Allum WH, Stenning SP, Thompson JN, Van de Velde CJ, Nicolson M, Scarffe JH, Lofts FJ, Falk SJ, Iveson TJ, Smith DB, Langley RE, Verma M, Weeden S, Chua YJ. Perioperative chemotherapy versus surgery alone for resectable gastroesophageal cancer. N Engl J Med 2006; 355(1): 11-20.

16. Cunningham $D$, Starling $N$, Rao $S$, Iveson $T$, Nicolson $M$, Coxon F, Middleton G, Daniel F, Oates J, Norman AR. Capecitabine and oxaliplatin for advanced esophagogastric cancer. N Engl J Med 2008; 358(1): 36-46.

17. Dank M, Zaluski J, Barone C, Valvere V, Yalcin S, Peschel C, Wenczl M, Goker E, Cisar L, Wang K, Bugat R. Randomized phase III study comparing irinotecan combined with 5 -fluorouracil and folinic acid to cisplatin combined with 5-fluorouracil in chemotherapy naive patients with advanced adenocarcinoma of the stomach or esophagogastric junction. Ann Oncol 2008; 19(8): 1450-7.

18. Dent DM, Werner ID, Novis B, Cheverton P, Brice P: Prospective randomized trial of combined oncological therapy for gastric carcinoma. Cancer 1979; 44(2): 385-91.

19. Eisenhauer EA, Therasse P, Bogaerts J, Schwartz LH, Sargent D, Ford R, Dancey J, Arbuck S, Gwyther S, Mooney M, Rubinstein L,
Shankar L, Dodd L, Kaplan R, Lacombe D, Verweij J. New response evaluation criteria in solid tumors: revised Recist guideline(version 1.1). Eur J Cancer 2009;45:228-47.

20. Estimativa. Incidência de Câncer no Brasil. INCA 2010.

21. Fink $U$, Schuhmacher $C$, Stein HJ, Busch R, Feussner $H$, Dittler $H J$, Helmberger A, Böttcher K, Siewert JR. Preoperative chemotherapy for stage III-IV gastric carcinoma: feasibility, response and outcome after complete resection. Br J Surg 1995; 82(9): 1248-52.

22. Gallardo-Rincon D, Onate-Ocana LF, Calderillo-Ruiz G: Neoadjuvant chemotherapy with P-ELF (cisplatin, etoposide, leucovorin, 5 -fluorouracil) followed by radical resection in patients with initially unresectable gastric adenocarcinoma: a phase II study. Ann Surg Oncol 2000; 7(1): 45-50.

23. Glimelius B, Ekström K, Hoffman K, Graf W, Sjödén PO, Haglund $U$, Svensson C, Enander LK, Linné T, Sellström $H$, Heuman R. Randomized comparison between chemotherapy plus best supportive care with best supportive care in advanced gastric cancer. Ann Oncol 1997; 8(2): 163-8.

24. Hermanek P, Wittekind C. The pathologist and the residual tumor( $R$ ) classification. Pathol Res Pract 1994;190:115-23.

25. Ishigami $S$, Natsugoe $S$, Nakajo $A$, Matsumoto $M$, Uenosono Y, Arigami T, Setoyama T, Arima H, Uchikado A, Kita Y, Sasaki K, Aikou T. Salvage gastrectomy following a combination of biweekly paclitaxel and S-1 for stage IV gastric cancer. J Gastrointest Surg 2008;12:1370-75.

26. Karnofsky DA, Burchenal JH. The clinical evaluation of chemotherapeutic agents in cancer. In: MacLeod CM (Ed), Evaluation of Chemotherapeutic Agents. Columbia Univ Press, 1949 P. 196.

27. Kelsen $D$, Karpeh $M$, Schwartz $G$, Gerdes $H$, Lightdale $C$, Botet J, Lauers G, Klimstra D, Huang $Y$, Saltz L, Quan V, Brennan M. Neoadjuvant therapy of high-risk gastric cancer: a phase II trial of preoperative FAMTX and postoperative intraperitoneal fluorouracilcisplatin plus intravenous fluorouracil. J Clin Oncol 1996; 14(6): 1818-28.

28. Kingston RD, Ellis DJ, Powell J, Brookes VS, Waterhouse JA, Hurst MD, Smith JA. The West Midlands gastric carcinoma chemotherapy trial: planning and results. Clin Oncol 1978; 4(1): 55-69.

29. Koizumi W, Narahara H, Hara T, Takagane A, Akiya T, Takagi M, Miyashita K, Nishizaki T, Kobayashi O, Takiyama W, Toh Y, Nagaie T, Takagi S, Yamamura Y, Yanaoka K, Orita H, Takeuchi M. S-1 plus cisplatin versus S-1 alone for first-line treatment of advanced gastric cancer (SPIRITS trial): a phase III trial. Lancet Oncol 2008; 9(3): 215-21.

30. Oken MM, Creech RH, Tormey DC. Toxicity and response criteria of the Eastern Cooperative Oncology Group. Am. J. Clin. Oncol. 1982;5 (6): 649-55.

31. Petrelli NJ, Winer EP, Brahmer J, Dubey S, Smith S, Thomas C, Vahdat LT, Obel J, Vogelzang N, Markman M, Sweetenham JW, Pfister D, Kris MG, Schuchter LM, Sawaya R, Raghavan D, Ganz PA, Kramer B. Clinical Cancer Advances 2009: major research advances in cancer treatment, prevention, and screening--a report from the American Society of Clinical Oncology. J Clin Oncol 2009; 27(35): 6052-69.

32. Plukker JT, Mulder NH, Sleijfer DT, Grond J, Verschueren RC: Chemotherapy and surgery for locally advanced cancer of the cardia and fundus: phase II study with methotrexate and 5-fluorouracil. $\mathrm{Br}$ J Surg 1991; 78(8): 955-8.

33. Rake MO, Mallinson CN, Cocking JB, Cwynarski MT, Fox CA, Wass VJ, Diffey BL, Jackson GA. Chemotherapy in advanced gastric cancer: a controlled, prospective, randomised multi-centre study. Gut 1979; 20(9): 797-801.

34. Saidi RF, ReMine SG, Dudrick PS, Hanna NN. Is There a Role for Palliative Gastrectomy in Patients with Stage IV Gastric Cancer? World J Surg 2006;30:21-27.

35. Schuhmacher CP, Fink U, Becker K, Busch R, Dittler HJ, Mueller J, Siewert JR. Neoadjuvant therapy for patients with locally advanced gastric carcinoma with etoposide, doxorubicin, and cisplatinum. Closing results after 5 years of follow-up. Cancer 2001; 91(5): 91827.

36. Szczepanik A, Kolodziejczyk P, Drabik G, Bucki K, Milanowski W. Cancer cells in bone marrow of gastric cancer patients during follow-up after radical resection - preliminary report. Poster 129, 8th International Gastric Cancer Congress, Abstract Book:134. 
37. Triazinate and platinum efficacy in combination with 5-fluorouracil and doxorubicin: results of a three-arm randomized trial in metastatic gastric cancer. Gastrointestinal Tumor Study Group. J Natl Cancer Inst 1988; 80(13): 1011-5.

38. Tsujinaka T, Fujitani K, Yang $H$, Kurokawa $Y$, Park B, Fukuda $H$, Noh S, Boku N, Bang Y, Sasako M, Lee J. A randomized controlled trial comparing gastrectomy plus Chemotherapy with chemotherapy alone in advanced gastric cancer with a single non-curable factor: JCOG 0705 and KGCA01. Abstract S64, 8th International Gastric Cancer Congress, Abstract Book:32.

39. Uenosono Y., Arigami T., Kozono T., Yanagita S., Hirata M., Arima H., Ishigami S., Aikou T., Natsugoe S. Circulating tumor cells in blood detected by CellSearch System are predictive factor of recurrences in gastric cancer. Abstract S57, 8th International Gastric Cancer Congress, Abstract Book:30.

40. Van Cutsem E, Moiseyenko VM, Tjulandin S, Majlis A, Constenla M, Boni C, Rodrigues A, Fodor M, Chao Y, Voznyi E, Risse ML, Ajani JA. Phase III study of docetaxel and cisplatin plus fluorouracil compared with cisplatin and fluorouracil as first-line therapy for advanced gastric cancer: a report of the V325 Study Group. J Clin Oncol 2006; 24(31): 4991-7.
41. Wagner AD, Grothe W, Haerting J, Kleber G, Grothey A, Fleig WE: Chemotherapy in advanced gastric cancer: a systematic review and meta-analysis based on aggregate data. J Clin Oncol 2006; 24(18): 2903-9.

42. Wilke H, Preusser P, Fink U, Gunzer U, Meyer HJ, Meyer J, Siewert $J R$, Achterrath W, Lenaz L, Knipp H. Preoperative chemotherapy in locally advanced and nonresectable gastric cancer: a phase II study with etoposide, doxorubicin, and cisplatin. J Clin Oncol 1989; 7(9): 1318-26.

43. Wittekind C, Compton CC, Greene FL, Sobin LH. TNM residual tumor classification revisited. Cancer 2002;94(9):2511-16.

44. Yano $M$, Shiozaki $H$ Inoue $M$, Tamura $S$, Doki Y,Yasuda $T$, Fujiwara Y, Tsujinaka T, Monden M. Neoadjuvant Chemotherapy followed by Salvage Surgery: Effect on survival of patients with primary noncurative gastric cancer. World J Surg 2002; 26:1155-59.

45. Xu RH, Teng KY: Progress in chemotherapy for advanced gastric cancer. Chin J Cancer 2009; 28(10): 1108-13. 